\title{
Dynamic parameter identification of tool-spindle interface based on RCSA and particle swarm optimization
}

\author{
Erhua Wang ${ }^{\mathrm{a}, \mathrm{c}}$, Bo Wu $\mathrm{Wu}^{\mathrm{b}, *}$, Youmin $\mathrm{Hu}^{\mathrm{a}}$, Shuzi Yang ${ }^{\mathrm{a}}$ and Yao Cheng ${ }^{\mathrm{a}}$ \\ a School of Mechanical Science and Engineering, Huazhong University of Science and Technology, Wuhan, Hubei, \\ China \\ ${ }^{\mathrm{b}}$ State Key Laboratory for Digital Manufacturing Equipment and Technology, Huazhong University of Science and \\ Technology, Wuhan, Hubei, China \\ ${ }^{\mathrm{c}}$ Mechanics and Electricity Department, Nanyang Institute of Technology, Nanyang, Henan, China
}

Received 7 February 2012

Revised 17 May 2012

\begin{abstract}
In order to ensure the stability of machining processes, the tool point frequency response functions (FRFs) should be obtained initially. By the receptance coupling substructure analysis (RCSA), the tool point FRFs can be generated quickly for any combination of holder and tool without the need of repeated measurements. A major difficulty in the sub-structuring analysis is to determine the connection parameters at the tool-holder interface. This study proposed an identification method to recognize the connection parameters at the tool-holder interface by using RCSA and particle swarm optimization (PSO). In this paper, the XHK machining center is divided into two components, which are the tool and the spindle assembly firstly. After that, the end point FRFs of the tool are achieved by mode superposition method. The end receptances of the spindle assembly with complicated structure are obtained by impacting test method. Through translational and rotational springs and dampers, the tool point FRF of the machining center is obtained by coupling the two components. Finally, PSO is adopted to identify the connection parameters at the tool-holder interface by minimizing the difference between the predicted and the measured tool point FRFs. Comparison results between the predicted and measured tool point FRFs show a good agreement and demonstrate that the identification method is valid in the identification of connection parameters at the tool-holder interface.
\end{abstract}

Keywords: Parameter identification, spindle dynamics, RCSA, particle swarm optimization (PSO)

\section{Introduction}

Regenerative chatter is a well-known machining problem that could result in unstable cutting process, poor surface quality and reduced material removal rate. In order to employ the total capacity of machining tools in production, researchers have done a lot of work on the prediction and suppression of chatter. Their work has made stability lobe diagrams as the main tool to identify the stable and unstable cutting zone in the machining process. As a function of the chip width and the spindle speed stability diagrams can be used to choose stable process parameters without losing productivity. Stability lobe diagrams could be acquired by using analytic [1-3] or numerical time-domain techniques [4]. Regardless of the approaches, the tool point FRF of a machine tool should be achieved firstly. In the orthogonal cutting process, the well-known relation for the limiting width of cut $\left(b_{\mathrm{lim}}\right)$ is given as

\footnotetext{
* Corresponding author: Bo Wu, State Key Laboratory for Digital Manufacturing Equipment and Technology, Huazhong University of Science and Technology, Wuhan 430074, Hubei, China. E-mail: bowu@mail.hust.edu.cn.
} 


$$
b_{\lim }=-\frac{1}{2 K_{t} z_{a} \operatorname{Re}[G(i \omega)]_{\min }}
$$

where $K_{t}$ is the cutting force coefficient in chip thickness direction, $z_{a}$ is the average number of teeth in cut, and $G(i \omega)$ refers to the cross FRF between the cutting force and the relative displacement in the direction normal to cut surface.

The tool point FRF is typically obtained by impacting test method [5-7]. The impacting force is applied on the tool tip and an accelerometer is placed at the same position for obtaining the force and acceleration signals. Then the tool point FRF can be achieved through a spectrum analyzer or modal testing software. However, for a different combination of holder and tool, the same test will be repeated since the system dynamics will change. Due to the large number of holder and tool combinations, these tests can prove time-consuming, and result in costly machining downtime. In order to minimize experimentation, Schmitz et al. [8,9] proposed a receptance coupling technique based on the fundamental sub-structuring analysis method. In the coupling technique, experimental or analytic FRFs of all components are coupled to obtain the receptance of the assembly [10-16].

The accuracy of the receptance coupling technique depends on accurate identification of the joint dynamics of the substructures at the assembly joint, and the FRFs of each substructure [12]. In many circumstances, the only variable portion of a machine tool is the overhang portion of the tool [16]. Therefore, the identification of connection parameters at the tool-holder interface is of utmost importance for obtaining the accurate tool point FRF. Through a series of linear transformation matrices, an identification method which is insensitive to measurement errors, was proposed to recognize the joint properties of a structure by using FRF data and other available knowledge [17]. As a general method of joint identification, it should be modified a lot to determine the connection parameters at the tool-holder interface. A finite element model was presented to find the stiffness and damping behavior between the tool and holder in thermal shrink fit connections, and the joint behavior was used to obtain the end FRFs of the tool-holder assembly [18]. It might be a preferred solution to deal with the problems of continuous interface between two substructures. An identification model was established to recognize the contact stiffness and damping at the interface of two substructures by using some optimization algorithms [19,20]. The identification approach might not work well for the tool with large aspect ratio, because the tool point FRF mainly depends on the tool dynamics. Based on the effect analysis of bearings and interface dynamics on the tool point FRF, it indicated that contact stiffness and damping values alter the frequencies and peak values of dominant vibration modes, respectively. According to its relevant mode, each contact parameter at the spindle-holder and tool-holder interfaces was achieved according to its relevant mode [21]. The research object is a given combination of spindle-holder-tool, so it is very difficult to be implemented in the practical machining center.

Based on RCSA and PSO, an identification approach is proposed to determine the connection parameters at the tool-holder interface in this paper. This study uses Euler-Bernoulli beam theory for calculating the end receptances of the tool by using mode superposition method. The end FRFs of the spindle assembly with complicated structure are obtained by impacting test method. Through translational and rotational springs and dampers, the two components are coupled to achieve the tool point FRF of the machining center. Finally, PSO is employed to determine the connection parameters at the tool-holder interface by minimizing the difference between the predicted and measured tool point FRFs. Henceforth, the paper is organized as follows. In Section 2, the end FRFs of the tool and the spindle assembly were respectively obtained by analytical and experimental methods, and they were coupled by RCSA method. In Section 3, PSO was employed to identify the connection parameters at the tool-holder interface. In Section 4, experimental verification and discussions were presented. The paper was concluded with a summary of contributions.

\section{Mathematical model}

\subsection{Component frequency response functions}

In order to obtain the connection parameters at the tool-holder interface, the machining center is divided into two components, which are $A$ (the tool) and $B$ (the spindle assembly) as illustrated in Fig. 1. Because of its linear characteristics, the free-free state end FRFs of the tool are calculated by Euler-Bernoulli beam theory. The analytical method, rather than impacting test, is employed due to these difficulties as follows. 


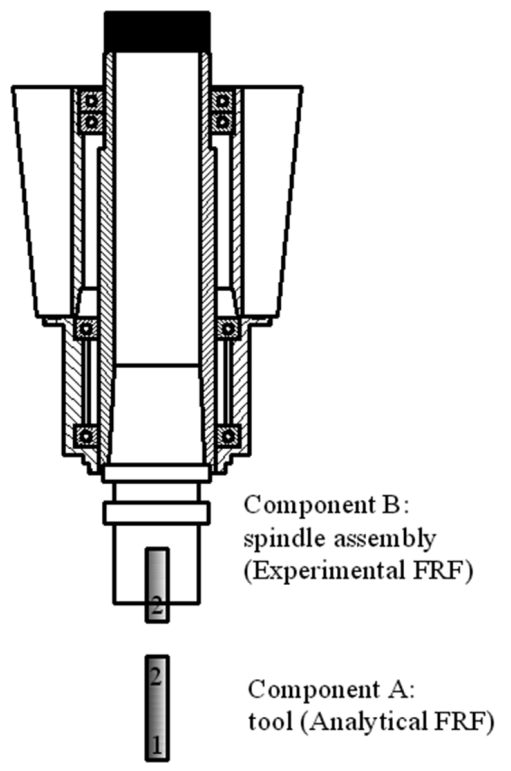

Fig. 1. Components of the tool and spindle assembly.

(1) The free-free state is difficult to realize in practice; (2) Lowmass, widebandwidth accelerometers typically do not perform well at low frequencies and zero frequency; (3) Rigid body mode may not be well represented in the measurements; (4) The responses of lowmass tools are easily affected by the accelerometers [9].

Whereas, the end FRFs of the spindle assembly are obtained by impacting test method.

The tool receptances are obtained based on the Euler-Bernoulli beam theory. The free motion equation of the tool is shown in Eq. (2).

$$
\rho \frac{\partial^{2} y(x, t)}{\partial t^{2}}+E I \frac{\partial^{4} y(x, t)}{\partial x^{4}}=q(x, t)
$$

where, $y(x, t)$ and $q(x, t)$ are the temporal displacement and the externally applied loads at any point along the beam, respectively. $\rho$ is the beam mass per unit length, $E$ is Young's modulus (homogeneity assumed), and $I$ is the second area moment of inertia.

Eigenvalue $\lambda_{r}$ of the $r$-th mode could be obtained from Eq. (2). By using these values, the dynamic transverse deflection can be expressed as

$$
\phi_{r}(x)=\cosh \frac{\lambda_{r} x}{L}+\cos \frac{\lambda_{r} x}{L}-\sigma_{r}\left(\sinh \frac{\lambda_{r} x}{L}+\sin \frac{\lambda_{r} x}{L}\right)
$$

The dynamic bending rotation can be expressed as

$$
\psi_{r}(x)=\phi_{r}(x)^{\prime}=\frac{\lambda_{r}}{L}\left[\sinh \frac{\lambda_{r} x}{L}-\sin \frac{\lambda_{r} x}{L}-\sigma_{r}\left(\cosh \frac{\lambda_{r} x}{L}+\cos \frac{\lambda_{r} x}{L}\right)\right]
$$

Here

$$
\sigma_{r}=\frac{\cosh \lambda_{r}-\cos \lambda_{r}}{\sinh \lambda_{r}-\sin \lambda_{r}}
$$

In order to obtain the uniform expressions of the tool end FRFs, the eigenfunctions should be normalized by the mass normalization, which satisfies the following orthogonality condition:

$$
\int_{x=0}^{L}\left\{U_{r}(x)\right\}^{T}[M]\left\{U_{s}(x)\right\} d_{x}=\left\{\begin{array}{l}
1, r=s \\
0, r \neq s
\end{array}\right.
$$




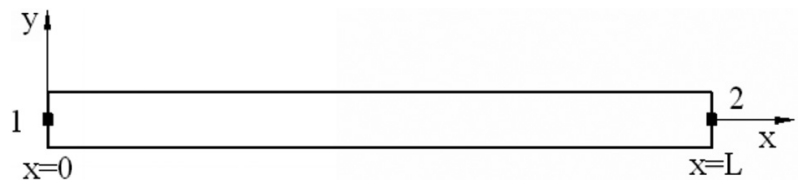

Fig. 2. Uniform Euler-Bernoulli beam with free end conditions

where

$$
\left\{U_{r}(x)\right\}=\left\{\begin{array}{l}
\phi_{r}(x) \\
\psi_{r}(x)
\end{array}\right\},[M]=\left[\begin{array}{cc}
\rho A & 0 \\
0 & \rho I
\end{array}\right]
$$

Since it is a free-free beam, the mass-normalized eigenfunctions of two rigid body modes are represented as

$$
\begin{aligned}
& \phi_{0}^{\text {trans }}(x)=\sqrt{\frac{1}{\rho A L}} \\
& \phi_{0}^{\text {rot }}(x)=\sqrt{\frac{12}{\rho A L^{3}}}\left(x-\frac{L}{2}\right)
\end{aligned}
$$

Here, $\phi_{0}^{\text {trans }}(x)$ and $\phi_{0}^{\text {rot }}(x)$ denote the eigenfunctions of translational and rotational rigid body modes, respectively.

The end FRFs are expressed as letters $H, N, L$ and $P$, and the definitions of them are as follows

$$
\begin{aligned}
& y_{j}=H_{j k} f_{k}, \quad y_{j}=L_{j k} m_{k}, \\
& \theta_{j}=N_{j k} f_{k}, \quad \theta_{j}=P_{j k} m_{k}
\end{aligned}
$$

where, $y$ and $\theta$ denote the translational and rotational deformations, respectively. And $f$ and $m$ represent the forces and the moments applied at the end points of interest, respectively.

Supposed that the structural damping is represented with a loss factor of $\gamma$, and the receptances defined above are given in Eqs (11)-(14) by mode superposition method [8,11].

$$
\begin{aligned}
H_{j k} & =\sum_{r=0}^{\infty} \frac{\phi_{r}\left(x_{j}\right) \phi_{r}\left(x_{k}\right)}{(1+i \gamma) \omega_{r}^{2}-\omega^{2}} \\
N_{j k} & =\sum_{r=0}^{\infty} \frac{\phi_{r}^{\prime}\left(x_{j}\right) \phi_{r}\left(x_{k}\right)}{(1+i \gamma) \omega_{r}^{2}-\omega^{2}} \\
L_{j k} & =\sum_{r=0}^{\infty} \frac{\phi_{r}\left(x_{j}\right) \phi_{r}^{\prime}\left(x_{k}\right)}{(1+i \gamma) \omega_{r}^{2}-\omega^{2}} \\
P_{j k} & =\sum_{r=0}^{\infty} \frac{\phi_{r}^{\prime}\left(x_{j}\right) \phi_{r}^{\prime}\left(x_{k}\right)}{(1+i \gamma) \omega_{r}^{2}-\omega^{2}}
\end{aligned}
$$

By substituting Eqs (3), (8) and (9) into Eqs (11)-(14), the end FRFs of points 1 and 2 can be achieved, respectively. Among them, the cross FRFs between points 1 and 2 are of the following form

$$
\begin{aligned}
& H_{12}=\frac{-1}{\rho A L \omega^{2}}+\frac{3}{\rho A L \omega^{2}}+\sum_{r=1}^{\infty} \frac{\phi_{r}\left(x_{1}\right) \phi_{r}\left(x_{1}\right)}{(1+i \gamma) \omega_{r}^{2}-\omega^{2}} \\
& N_{12}=\frac{-6}{\rho A L^{2} \omega^{2}}+\sum_{r=1}^{\infty} \frac{\phi_{r}^{\prime}\left(x_{1}\right) \phi_{r}\left(x_{1}\right)}{(1+i \gamma) \omega_{r}^{2}-\omega^{2}} \\
& L_{12}=\frac{-6}{\rho A L^{2} \omega^{2}}+\sum_{r=1}^{\infty} \frac{\phi_{r}\left(x_{1}\right) \phi_{r}^{\prime}\left(x_{1}\right)}{(1+i \gamma) \omega_{r}^{2}-\omega^{2}}
\end{aligned}
$$




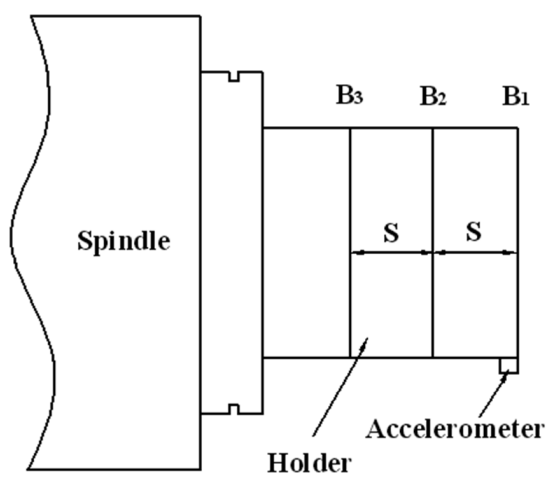

Fig. 3. Receptance identification of the spindle assembly.

$$
P_{12}=\frac{-12}{\rho A L^{3} \omega^{2}}+\sum_{r=1}^{\infty} \frac{\phi_{r}^{\prime}\left(x_{1}\right) \phi_{r}^{\prime}\left(x_{1}\right)}{(1+i \gamma) \omega_{r}^{2}-\omega^{2}}
$$

The remaining 12 FRFs of the tool can be obtained in a similar manner. The receptance matrix of the tool is expressed as follows

$$
[T]=\left[\begin{array}{ll}
T_{11} & T_{12} \\
T_{21} & T_{22}
\end{array}\right]=\left[\begin{array}{llll}
H_{11} & L_{11} & H_{12} & L_{12} \\
N_{11} & P_{11} & N_{12} & P_{12} \\
H_{21} & L_{12} & H_{22} & L_{22} \\
N_{21} & P_{21} & N_{22} & P_{22}
\end{array}\right]
$$

The direct FRFs of the spindle assembly are acquired by impacting test method. The impacting test is performed at locations $B_{1}, B_{2}$, and $B_{3}$ to obtain the direct FRF $H_{B 1 B 1}$, as well as the cross FRFs $H_{B 1 B 2}$ and $H_{B 1 B 3}$. Then a second order backward finite difference method is employed to obtain $L_{B 1 B 1}$ and $P_{B 1 B 1}$ [13].

$$
\begin{aligned}
L_{B 1 B 1} & =\frac{3 H_{B 1 B 1}-4 H_{B 1 B 2}+H_{B 1 B 3}}{2 S} \\
P_{B 1 B 1} & =\frac{\theta_{1}}{M_{1}}=\frac{F_{1}}{X_{1}} \frac{X_{1}}{M_{1}} \frac{\theta_{1}}{F_{1}}=\frac{L_{11}^{2}}{H_{11}}
\end{aligned}
$$

By reciprocity, $N_{33}$ can be set equal to $L_{33}$. So the direct receptances of the spindle assembly can be expressed as follows

$$
\left[S A_{11}\right]=\left[\begin{array}{ll}
H_{B 1 B 1} & L_{B 1 B 1} \\
N_{B 1 B 1} & P_{B 1 B 1}
\end{array}\right]
$$

The end FRFs of the spindle assembly are obtained by impacting test method, and the impacting test scheme is shown in Fig. 4. An accelerometer attached to the holder is used to obtain the acceleration signals excited by the impulse hammer at the locations $B_{1}, B_{2}$ and $B_{3}$. The acceleration and excitation force signals are acquired and the direct end FRFs of the spindle assembly are obtained by using LMS analysis software.

\subsection{Coupling of components}

After obtaining the end FRFs of each component, RCSA is used to achieve the tool point FRF of the coupled system as shown in Fig. 5. Here, $k_{y}$ and $c_{y}$ are the translational stiffness and damping at the tool-holder interface respectively. And $k_{\theta}$ and $c_{\theta}$ are the rotational stiffness and damping at the tool-holder interface, respectively. The interface dynamics between the holder and the tool can be expressed in Eq. (23).

$$
\left[K_{h t}\right]=\left[\begin{array}{cc}
k_{y}+i \omega c_{y}^{s h} & 0 \\
0 & k_{\theta}+i \omega c_{\theta}^{s h}
\end{array}\right]
$$

The tool receptances of the machining center can be coupled with the spindle assembly, and the direct FRF at the tool tip is obtained by Eq. (24) [11].

$$
\left[H_{11}\right]=\left[T_{11}\right]-\left[T_{12}\right]\left[\left[T_{22}\right]+\left[K_{h t}\right]^{-1}+\left[S A_{11}\right]\right]^{-1}\left[T_{21}\right]
$$




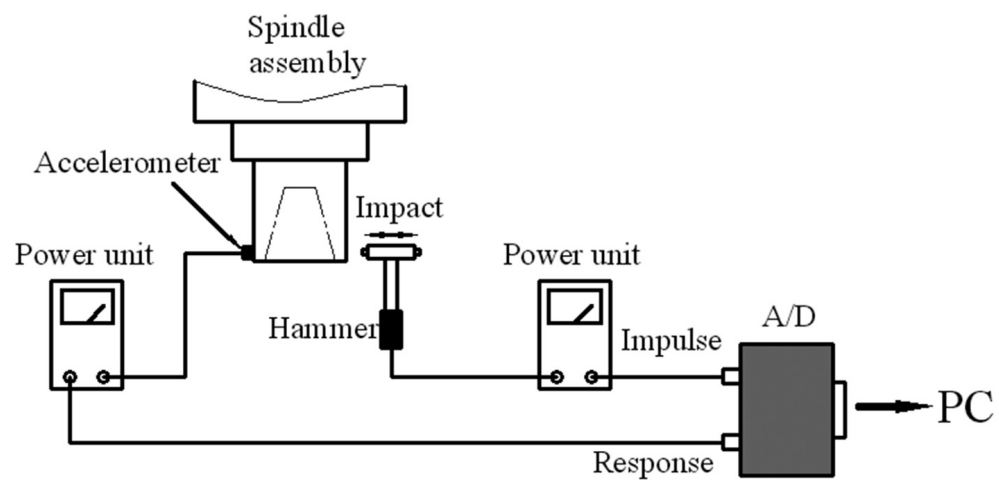

Fig. 4. Impacting test scheme of the spindle assembly.

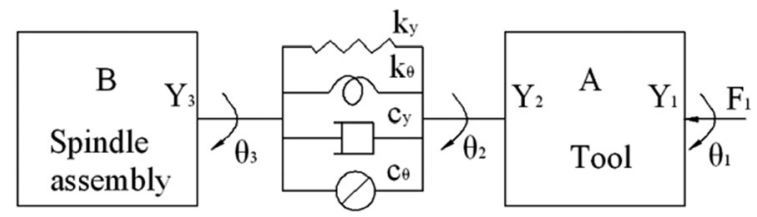

Fig. 5. Coupling model of the spindle-holder-tool assembly

\section{Parameter identification of the holder-tool interface}

In the direct tool point FRF Eq. (24), $k_{y}, c_{y}, k_{\theta}$ and $c_{\theta}$ are all unknows to be recognized. In order to improve the identification accuracy, PSO is adopted to determine the connection parameters at the tool-holder interface. These parameters can be represented as depicted in Eq. (25).

$$
\alpha=\left[k_{y}, c_{y}, k_{\theta}, c_{\theta}\right]
$$

When $\omega=\omega_{j}$, the predicted tool point FRF is $H_{11}\left(\omega_{j}\right)$, simplified as $H_{11}$. Meanwhile the measured FRF is $\tilde{H}_{11}\left(\omega_{j}\right)$, simplified as $\tilde{H}_{11}$. If $\omega_{j}=\omega_{1}, \omega_{2}, \ldots, \omega_{m}$, there are $m$ frequency measurement points, and an error vector $E$ can be constructed by Eq. (26)

$$
E=H_{11}(\alpha)-\tilde{H}_{11}
$$

In Eq. (26), $E$ is the nonlinear function of the vector $\alpha$ to be identified. The total variance $J(\alpha)$ is shown by Eq. (27).

$$
J(\alpha)=E^{H} E=\left\{H_{11}(\alpha)-\tilde{H}_{11}\right\}^{T}\left\{H_{11}(\alpha)-\tilde{H}_{11}\right\}
$$

The identification problem can be solved by the means of some optimization algorithms, such as Newton Method, Conjugate Gradient Method, Genetic Algorithm (GA), PSO, and et al. Among them, PSO is a bionic algorithm developed by Kennedy and Eberhart [22]. One of the advantages of the PSO algorithm compared with other evolutionary algorithms is that it can be easily programmed, which means no encoding or decoding processes are needed as they are in GA [23]. Therefore, PSO is chosen as the optimization algorithm to recognize the connection parameters at the tool-holder interface.

The PSO technique appeared as a promising algorithm for handling the optimization problems [24-26]. It is a population-based search algorithm for modeling a swarm of particles (bees or birds et. al) looking for food. When they are looking for food, each particle in the swarm is moving to the best position with a velocity from its previous position. There are three segments affecting the velocity of a particle, and they are inertial momentum, cognitive and social segment, respectively. Among them, the inertial segment denotes the inertial behavior of the particle to move in the previous direction, the cognitive segment denotes the memory of the particle about its previous best position, and the social component denotes the memory of the particle about the best position in the swarm. 


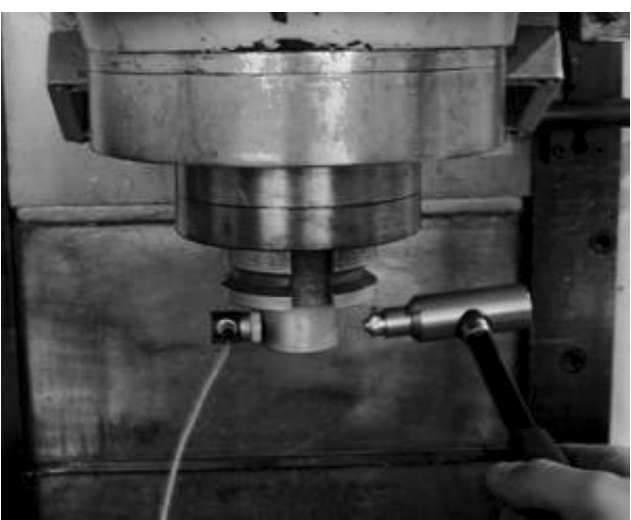

(a) Impacting test of the spindle assembly

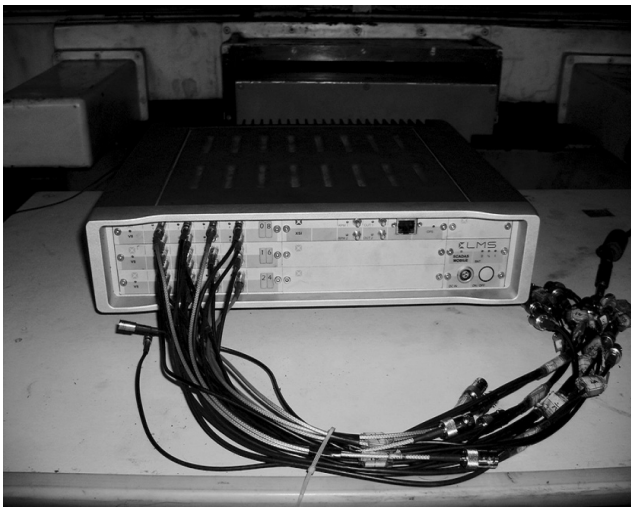

(c) LMS data acquisition system

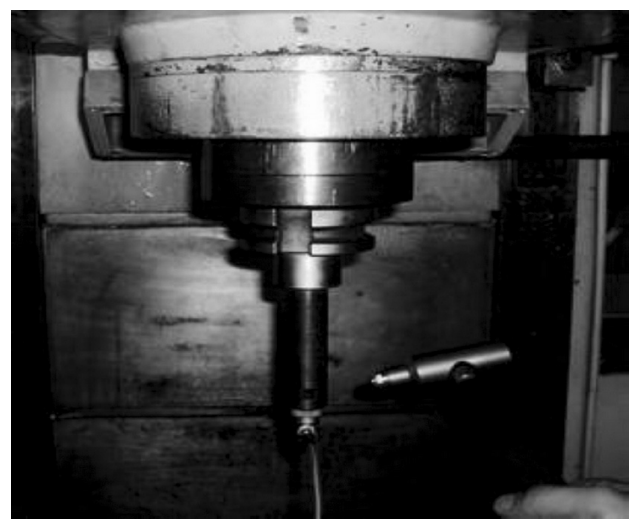

(b) Impacting test of the tool tip

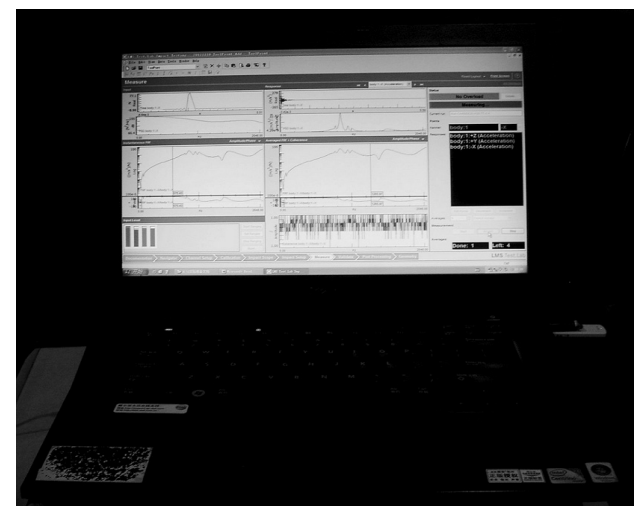

(d) Data acquisition and analysis

Fig. 6. Photographs of the experiment scene.

The global best position is represented by $P_{g}=\left(p_{g 1}, p_{g 2}, \ldots p_{g m}\right)$, while the velocity of the ith particle is represented by $V_{i}=\left(v_{i 1}, v_{i 2}, \ldots, v_{i m}\right)$. The modified velocity and position of each particle can be calculated according to the following equations:

$$
\begin{aligned}
v_{i}^{k+1} & =w v_{i}^{k}+c_{1} r_{1}\left(p_{i}-\alpha_{i}^{k}\right)+c_{2} r_{2}\left(p_{g}^{k}-\alpha_{i}^{k}\right) \\
\alpha_{i}^{k+1} & =\alpha_{i}^{k}+v_{i}^{k+1}
\end{aligned}
$$

where, $c_{1}$ and $c_{2}$ are two positive constants known as acceleration coefficients; $w$ is the inertia weight which controls the exploration and exploitation ability of the swarm; and $r_{1}$ and $r_{2}$ are two random numbers in the range [0,1].

In the initial iterations, the particles have higher exploration ability in the global search region. As the iterations evolve, the particles have more strong exploitation ability in the local search region. If exploration is stressed, convergence may not be reached. If exploitation is stressed, premature convergence can appear in some local region which is far from the global optimization. Proper balance between exploration and exploitation is of fundamental importance for successful searches, assuring simultaneously global search characteristics and high precision of the final results. In order to increase the exploration during the initial search iterations and the exploitation during the final steps of the search, inertia weight $w$ and acceleration coefficients $c_{1}$ and $c_{2}$ can be adjusted as follows

$$
\begin{aligned}
& w_{i}=w_{\max }-\frac{w_{\max }-w_{\min }}{\text { iter }_{\max }} \times \text { iter } \\
& c_{1}=c_{1 i}+\frac{c_{1 f}-c_{1 i}}{\text { iter }_{\max }} \times \text { iter } \\
& c_{2}=c_{2 i}+\frac{c_{2 f}-c_{2 i}}{\text { iter }_{\max }} \times \text { iter }
\end{aligned}
$$



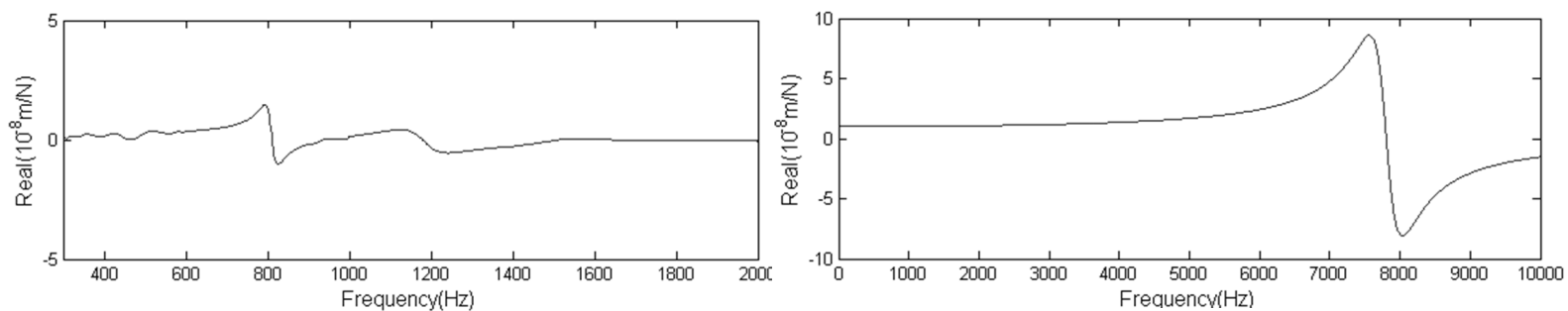

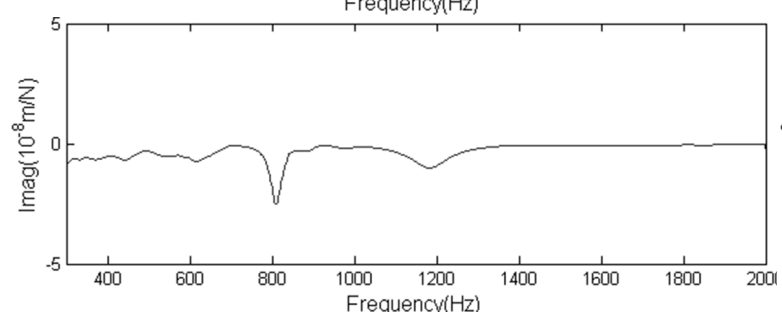

(a)

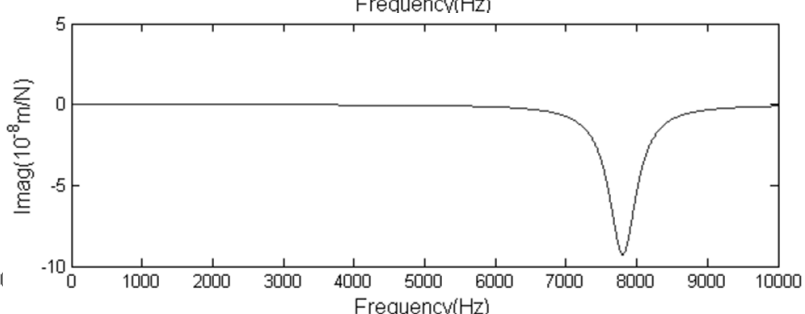

(b)

Fig. 7. Frequency response functions of the spindle assembly and the tool. (a) FRF of the spindle assembly measured by impacting tests, (b) FRF of the tool identified by analytical method.

where $w_{\max }$ and $w_{\min }$ denote the maximum and minimum values of the inertia weight, respectively. $c_{1 i}, c_{1 f}$ and $c_{2 i}, c_{2 f}$ are the initial and final values of acceleration coefficients $c_{1}$ and $c_{2}$, respectively. iter $\max$ is the maximum iteration number, and iter is the current iteration number. In this paper, $c_{1 i}=2.5, c_{1 f}=0.5, c_{2 i}=0.5, c_{2 f}=2.5$, iter $_{\max }=50$, the number of particles $N=30$, and the number of search dimensions $M=4$.

The iterative steps for PSO algorithm can be described as follows:

(1) Each particle can be expressed as $\alpha^{k}=\left[k_{y}^{k}, c_{y}^{k}, k_{\theta}^{k}, c_{\theta}^{k}\right]$. Initial positions and velocities for all the particles are designated in Eq. (33). The current position of each particle is generated and the best position of the ith particle is stored as $p_{i}=\left(p_{i 1}, p_{i 2}, \ldots, p_{i m}\right)$. The current best position within the global particle swarm is stored as $p_{g}=\left(p_{g 1}, p_{g 2}, \ldots, p_{g m}\right)$.

$$
\begin{aligned}
\alpha_{i j}^{0} & =\alpha_{j \min }+\operatorname{rand}(i, j)\left(\alpha_{j \max }-\alpha_{j \min }\right) \\
v_{i j}^{0} & =v_{j \min }+\operatorname{rand}(i, j)\left(v_{j \max }-v_{j \min }\right) \\
v_{j \max } & =-v_{j \min }=x_{j \max }-x_{j \min } \\
i & =1,2, \ldots, N ; \quad j=1,2, \ldots, M
\end{aligned}
$$

(2) The new position for each particle is generated according to Eqs (28) and (29).

(3) The value of objective function is calculated for the new position of each particle. If a particle reaches a better position, its $p_{i}$ value is replaced by the current value.

(4) A $p_{g}$ value is chosen from the new set of $p_{i}$ values. If the new $p_{g}$ value is better than the previous one, the previous $p_{g}$ value will be replaced by the new one.

(5) Repeat Steps (2)-(4) until the predetermined number of iterations is reached.

\section{Experiment verification}

In this case study, the tool material is steel with mass density $7800 \mathrm{~kg} / \mathrm{m}^{3}$, Young's modulus $E=200 \mathrm{Gpa}$ and Poisson's ratio $v=0.3$. In addition, the material loss factor is assumed to be $\gamma=0.003$. The direct and cross FRFs of the tool are calculated by the related analytical formulas presented above. The direct FRFs of the spindle assembly are obtained by impacting test shown in Fig. 6(a).

The analytical and measured results are shown in Fig. 7. Figure 7(a) shows the translational part of this response. The spindle assembly has two dominant modes at 812 and $1195 \mathrm{~Hz}$, respectively. Figure 7(b) shows the analytical FRF of the tool. The first elastic mode of the tool occurs approximately at $7805 \mathrm{~Hz}$. 
Table 1

Identified holder-tool interface parameters

\begin{tabular}{cccc}
\hline$k_{y}(N / m)$ & $k_{\theta}(N . m / r a d)$ & $c_{y}(N . m . s / r a d)$ & $c_{\theta}(N . s / m)$ \\
\hline $5.6 \mathrm{e} 7$ & $6.2 \mathrm{e} 6$ & 91 & 33 \\
\hline
\end{tabular}
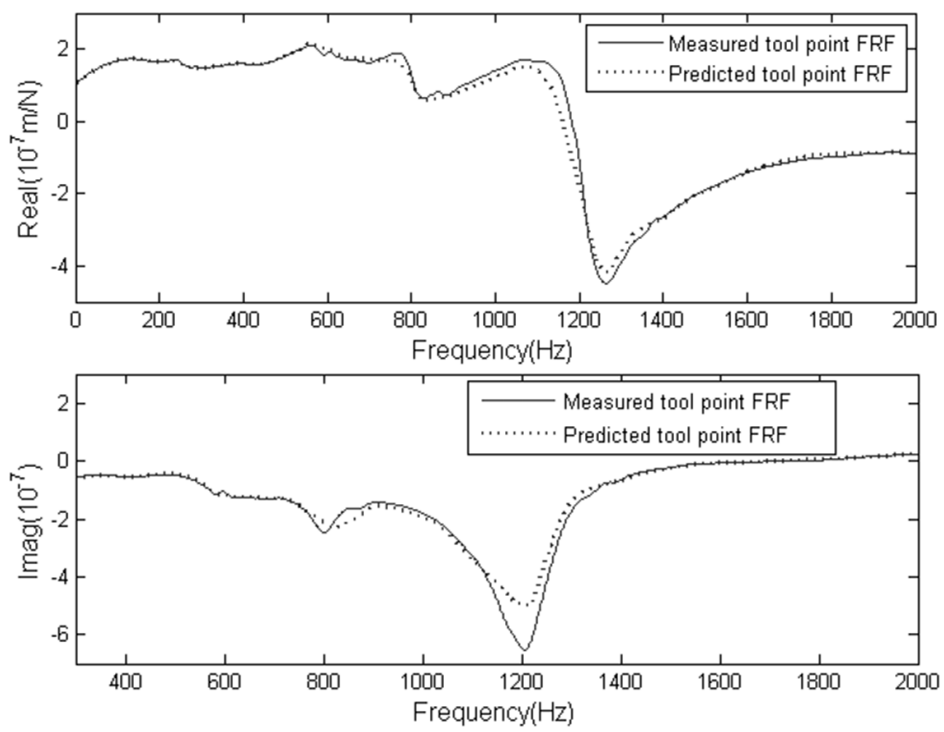

Fig. 8. Predicted and measured tool point FRF of the XHK machining center.

The tool point FRF of the machining center is obtained by coupling the end FRFs of component A and B based on RCSA. On the basis of PSO above, the connection parameters at the tool-holder interface are determined. The identified interface parameters are shown in Table 1.

To verify the identification approach, an impacting test is performed on the XHK machining center produced by Huazhong NC Company. The predicted tool point FRF is compared with the measured one. And the comparison results between the predicted and measured tool point FRFs are shown in Fig. 8.

In Fig. 8, it can be observed that the predicted tool point FRF shows a good agreement with the measured one generally. Therefore, it can be concluded that the proposed identification method is valid in the recognition of connection parameters at the tool-holder interface.

\section{Conclusion}

In this paper, an identification model is built to identify the connection parameters at the tool-holder interface based on RCSA and PSO. The machining center is divided into two components which are the tool and the spindle assembly. The end FRFs of the tool are calculated by the Euler-Bernoulli beam theory. Meanwhile, the end receptances of the spindle assembly are obtained experimentally. Then the two components are coupled through the translational and rational spring and damping elements. By minimizing the difference between the predicted and the measured tool point FRFs, PSO is employed to identify the connection parameters at the tool-holder interface. Finally, comparison results between the predicted and the measured tool point FRFs verify the proposed identification method and its capability in identifying the connection parameters at the tool-holder interface. After obtaining each connection parameter at the tool-holder interfaces, the tool point FRFs can be generated quickly for any combination of holder and tool without the need for repeated measurements. 


\section{Acknowledgments}

The work is supported by the National Key Basic Research Program of China (No. 2011CB706803) and National Natural Science Foundation of China (NO. 51175208)

\section{References}

[1] Y. Altintas and E. Budak, Analytical prediction of stability lobes in milling, CIRP Ann 44(1) (1995), 357-362.

[2] Y. Altintas and P. Lee, A general mechanics and dynamics model for helical end mills, CIRP Ann 45(1) (1996), 59-64.

[3] E. Budak and Y. Altintas, Analytical prediction of chatter stability in milling-Part I: General formulation; PartII: Application to common milling systems, Transactions of ASME, Journal of Dynamic Systems, Measurement and Control 120 (1998), 22-36.

[4] S. Smith and J. Tlusty, An overview of modeling and simulation of the milling process, Transactions of ASME, Journal of Engineering for Industry 113(2) (1991), 169-175.

[5] E.B. Kivanc, E. Budak et al., Structural modeling of end mills for form error and stability analysis, International Journal of Machine Tools and Manufacture 44(11) (2004), 1151-1161.

[6] E. Budak et al., Analytical models for high performance milling, Part I: Cutting forces, structural deformations and tolerance integrity, International Journal of Machine Tools and Manufacture 46(12-13) (2006), 1478-1488.

[7] E. Budak et al., Analytical models for high performance milling, Part II: Process dynamics and stability, International Journal of Machine Tools and Manufacture 46 (2006), 1489-1499.

[8] T. Schmitz, Predicting high-speed machining dynamics by substructure analysis, CIRP Ann 49(1) (2000), 303-308.

[9] T. Schmitz, M. Davies and M. Kennedy, Tool point frequency response prediction for high-speed machining by RCSA, Journal of Manufacturing Science and Engineering 123 (2001), 700-707.

[10] T. Schmitz, M. Davies, K. Medicus and J. Synder, Improving high-speed machining material removal rates by rapid dynamic analysis, Annals of the CIRP 50(1) (2001), 263-268.

[11] T. Schmitz and T. Burns, Receptance coupling for high-speed machining dynamics prediction, in: Proceedings of the 21st International Modal Analysis Conference 36 (February 2003), Kissimmee, FL (on CD).

[12] S.S. Park, Y. Altintas and M. Movahhedy, Receptance coupling for end mills, International Journal of Machine Tools \& Manufacture $\mathbf{4 3}$ (2003), 889-896.

[13] T. Schmitz and G.S. Duncan, Three-component receptance coupling substructure analysis for tool point dynamics prediction, Journal of Manufacturing Science and Engineering 127 (2005), 781-790.

[14] A. Ertürk, H.N. Özgüven and E. Budak, Analytical modeling of spindletool dynamics on machine tools using Timoshenko beam model and receptance coupling for the prediction of tool point FRF, International Journal of Machine Tools \& Manufacture 46 (2006), 1901-1912.

[15] S. Filiz, C.H. Cheng etc., An improved toolholder model for RCSA tool-point frequency response prediction, Precision Engineering 33 (2009), 26-36.

[16] H. Ahmadian and M. Nourmohammadi, Tool point dynamics prediction by a three-component model utilizing distributed joint interfaces, International Journal of Machine Tools \& Manufacture 50 (2010), 998-1005.

[17] Y. Ren and C.F. Beards, Identification of joint properties of a structure using FRF data, Journal of Sound and Vibration 186(4) (1995), 567-587.

[18] T.L. Schmitz, K. Powell, D. Won etc., Shrink fit tool holder connection stiffness/damping modeling for frequency response prediction in milling, International Journal of Machine Tools \& Manufacture 47 (2007), 1368-1380.

[19] M.R. Movahhedy and J.M. Gerami, Prediction of spindle dynamics in milling by sub-structure coupling, International Journal of Machine Tools \& Manufacture 46 (2006), 243-251.

[20] M. Namazi, Y. Altintas, T. Abe and N. Rajapaks, Modeling and identification of tool holderspindle interface dynamics, International Journal of Machine Tools \& Manufacture 47 (2007), 1333-1341.

[21] O. Özọhin, A. Ertürk, H.N. Özgüven and E. Budak, A closed-form approach for identification of dynamical contact parameters in spindle-holder-tool assemblies, International Journal of Machine Tools \& Manufacture 49 (2009), 25-35.

[22] J. Kennedy and R.C. Eberhart, Particle swarm optimization, in: Proceedings of IEEE International Conference in Neural Networks, 1995, pp. 1942-1948.

[23] T.Y. Chen and T.M. Chi, On the improvements of the particle swarm optimization algorithm, Advances in Engineering Software 41 (2010), 229-239.

[24] P.J. Angeline, Using selection to improve particle swarm optimization, IEEE International Conference on Evolutionary Computation (1998), 84-89.

[25] R.C. Eberhart and Y. Shi, Particle swarm optimization: Developments applications and resourses, in: Proceedings Congress on Evolutionary Computation, IEEE service, NJ, Seoul, Korea, 2001.

[26] S.N. Deepa and G. Sugumaran, Model order formulation of a multivariable discrete system using a modified particle swarm optimization approach, Swarm and Evolutionary Computation (2011), 1-9. 

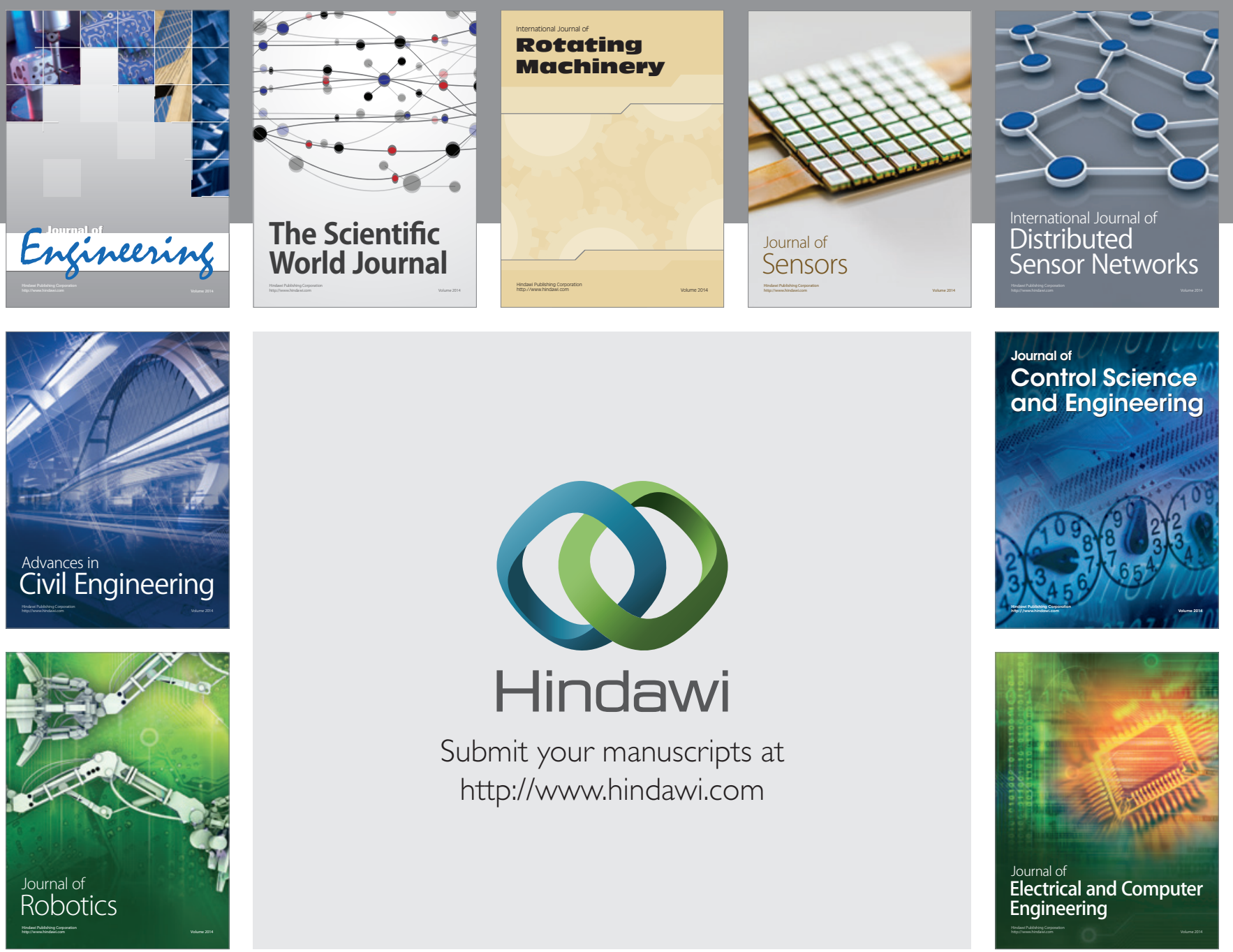

Submit your manuscripts at

http://www.hindawi.com
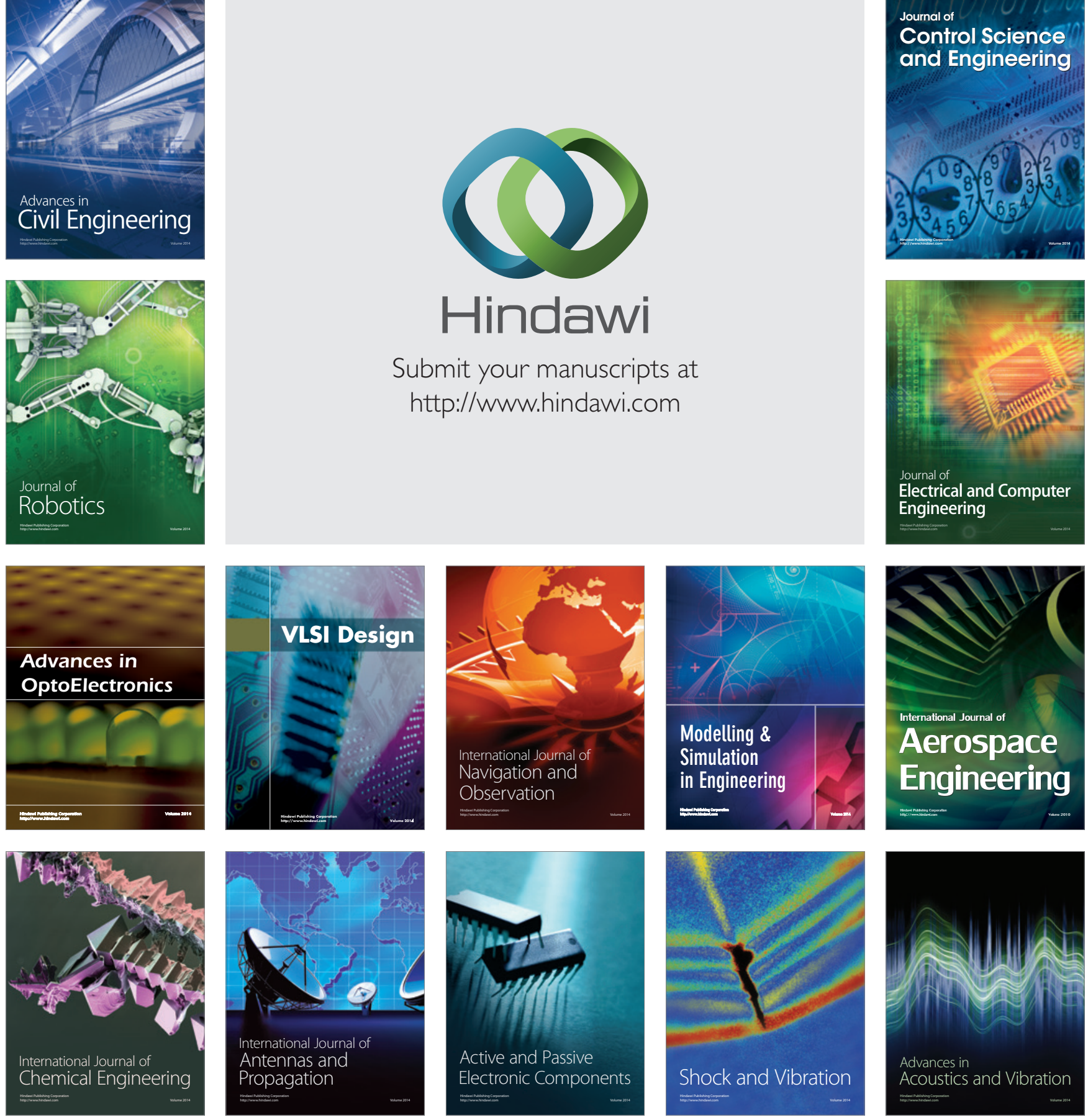